\title{
A novel GATA6 variant in a boy with neonatal diabetes and diaphragmatic hernia: a familial case with a review of the literature
}

\author{
Gaisl, Odile ; Konrad, Daniel ; Joset, Pascal ; Lang-Muritano, Mariarosaria
}

\begin{abstract}
GATA6 gene variants come along with possible features such as pancreas agenesis/hypoplasia, neonatal diabetes and congenital heart defect. Congenital hypothyroidism, and hepatobiliary and gut abnormalities are also detectable. Children with congenital heart defects and neonatal diabetes were already described in 1970 . GATA6 variants can be due to de novo variants or due to inherited variants. To date, 11 cases due to an inherited variant have been described. Herein we present a novel heterozygous GATA6 variant (c.1291C > T p. [Gln431*]) in a boy with transient neonatal diabetes, diaphragmatic hernia, congenital heart defect and early-onset scoliosis. The same variant was also present in the mother. At the age of 3 years, a random evaluation revealed a hemoglobin A1c (HbA1c) level of $7.8 \%(62 \mathrm{mmol} / \mathrm{mol})$ without any diabetes-related symptoms. He was started on insulin therapy and HbA1c normalized. A short review of the literature of hereditary cases of the GATA6 variant revealed the variable phenotypic spectrum and showed that patients with a mild phenotype are likely to have children with a more severe phenotype.
\end{abstract}

DOI: https://doi.org/10.1515/jpem-2019-0057

Posted at the Zurich Open Repository and Archive, University of Zurich ZORA URL: https://doi.org/10.5167/uzh-178436

Journal Article

Published Version

Originally published at:

Gaisl, Odile; Konrad, Daniel; Joset, Pascal; Lang-Muritano, Mariarosaria (2019). A novel GATA6 variant in a boy with neonatal diabetes and diaphragmatic hernia: a familial case with a review of the literature. Journal of Pediatric Endocrinology Metabolism, 32(9):1027-1030.

DOI: https://doi.org/10.1515/jpem-2019-0057 


\title{
Case Report
}

\author{
Odile Gaisl*, Daniel Konrad, Pascal Joset and Mariarosaria Lang-Muritano
}

\section{A novel GATA6 variant in a boy with neonatal diabetes and diaphragmatic hernia: a familial case with a review of the literature}

https://doi.org/10.1515/jpem-2019-0057

Received January 29, 2019; accepted May 18, 2019; previously published online July 4, 2019

\begin{abstract}
GATA6 gene variants come along with possible features such as pancreas agenesis/hypoplasia, neonatal diabetes and congenital heart defect. Congenital hypothyroidism, and hepatobiliary and gut abnormalities are also detectable. Children with congenital heart defects and neonatal diabetes were already described in 1970. GATA6 variants can be due to de novo variants or due to inherited variants. To date, 11 cases due to an inherited variant have been described. Herein we present a novel heterozygous GATA6 variant (c.1291C > T p. [Gln431*]) in a boy with transient neonatal diabetes, diaphragmatic hernia, congenital heart defect and early-onset scoliosis. The same variant was also present in the mother. At the age of 3 years, a random evaluation revealed a hemoglobin $\mathrm{A}_{1 \mathrm{c}}\left(\mathrm{HbA}_{1 \mathrm{c}}\right)$ level of $7.8 \%$ (62 $\mathrm{mmol} / \mathrm{mol})$ without any diabetes-related symptoms. He was started on insulin therapy and $\mathrm{HbA}_{1 c}$ normalized. A short review of the literature of hereditary cases of the GATA6 variant revealed the variable phenotypic spectrum and showed that patients with a mild phenotype are likely to have children with a more severe phenotype.
\end{abstract}

Keywords: congenital heart defect; diaphragmatic hernia; GATA6; hereditary; neonatal diabetes.

*Corresponding author: Odile Gaisl, Department of Pediatric Endocrinology and Diabetology, and Children's Research Centre, University Children's Hospital, Steinwiesstrasse 75, 8032 Zürich, Switzerland, Phone: +0041 4426684 23, Fax: +0041 4426679 83, E-mail: odile.gaisl@kispi.uzh.ch. https://orcid.org/0000-00029158-4368

Daniel Konrad and Mariarosaria Lang-Muritano: Department of Pediatric Endocrinology and Diabetology, and Children's Research Centre, University Children's Hospital, Zürich, Switzerland

Pascal Joset: Institute of Medical Genetics, University of Zurich, Schlieren-Zurich, Switzerland

\section{Introduction}

GATA6 is a zinc finger transcription factor that is important for the development of the cardiac and gastrointestinal systems and was identified for the first time in 1994 [1]. Already in 1970, children with congenital heart defects and neonatal diabetes were described in whom a GATA6 defect was identified subsequently [2]. Possible features of a GATA6 gene variant are pancreas agenesis, neonatal diabetes and congenital heart defect. Neonatal diabetes is a form of diabetes with onset in the first 6 months of life. It can be permanent or in about $50 \%$ of the cases, transient [3-5]. A high number of patients (about 50\%) have a relapse of diabetes in adolescence [4, 6]. Congenital hypothyroidism, and hepatobiliary and gut abnormalities (gallbladder agenesis, biliary atresia) have been described as well in patients with GATA6 $[7,8]$.

GATA6 variants can be due to de novo variants or due to inherited variants [8-12]. The aim of this study was to describe the novel hereditary GATA6 variant in our patient and his mother, and to present a short review of the literature with so-far described hereditary cases of the GATA6 variant.

\section{Case report}

A term-born boy, small for gestational age (39 weeks of gestation, $1970 \mathrm{~g}$ (-3.25 standard deviation [SD]), developed severe hyperglycemia up to $100 \mathrm{mmol} / \mathrm{L}$ within the first day of life. He was treated with intravenous insulin. Diabetes resolved after 5 days and insulin supplementation could be stopped. In addition to diabetes, the boy presented with diaphragmatic hernia and a complex congenital heart defect (high-grade stenosis of the left pulmonary artery, ventricular septal defect [VSD], persistent ductus arteriosus and atrial septal defect [ASD]). The transient neonatal diabetes was interpreted as a consequence of pancreas compression due 
to the diaphragmatic hernia. Hernia surgery was successful. At the age of 1 year, the boy presented with an early-onset scoliosis, which was treated with a corset. At the age of 3 years, a random evaluation revealed a hemoglobin $\mathrm{A}_{1 \mathrm{c}}\left(\mathrm{HbA}_{1 \mathrm{c}}\right)$ level of $7.8 \%(62 \mathrm{mmol} / \mathrm{mol})$ without any diabetes-related symptoms. Autoantibodies to insulin, glutamic acid decarboxylase, $\beta$ islet cells and zinc transporter-8 were negative. Exocrine pancreas insufficiency was excluded. Repeated abdominal ultrasonography revealed a small pancreas with reduced size of the head and tail, and no gallbladder could be found. He was started on single insulin injection with degludec (2.5 IE) and a loosened diet without strict counting of carbohydrates. Under such regimen, the $\mathrm{HbA}_{1 \mathrm{c}}$ value decreased to $6.2 \%$ (44 mol/mol). Growth was following the 25th-50th percentile, below the range for his target height.

His mother developed an autoantibody-negative diabetes at the age of 25 years and had a history of a complex congenital heart defect (ASD, VSD, hypoplastic aortic arch, bicuspid aortic valve [BAV], stenosis of the right pulmonary artery and persistent ductus arteriosus). Because of primary sterility, in vitro fertilization (IVF) was successfully performed and resulted in the mother's pregnancy with the aforementioned boy. Genetic investigations were performed in both the boy and his mother.

For molecular analysis, genomic DNA was extracted from ethylenediaminetetraacetic acid (EDTA) blood of the patient. Whole exome sequencing (WES) was performed using the Agilent (Santa Clara, CA, USA) SureSelectXT Kit (V6) with paired-end sequencing (HiSeq SBS Kit v4, 125 Fwd-125 Rev, Q30-value: 92.2) on a HiSeq System (Illumina Inc., San Diego, CA, USA). Raw fastQ files were aligned to the hy19 reference genome using NextGene (Softgenetics, LLC, State College, PA, USA). The average depth of coverage was $163 \mathrm{x}$, and about $97 \%$ of the targeted bases were assessed by $\geq 20$ independent sequence reads. Variants observed in at least $16 \%$ of reads with a sufficient quality level and with a minor allele frequency $\leq 2 \%$ were filtered against genes associated with diabetes and investigated for deleterious in silico effects. The GATA6 (NM_005257.5) variant from the WES approach was confirmed in the index and his mother after polymerase chain reaction (PCR) amplification by Sanger sequencing using an ABI Genetic Analyzer 3730 (Applied Biosystems, Foster City, CA, USA).

In both the patient and his mother, an identical heterozygous GATA6 gene variant (c.1291C > T p.[Gln431*]) was identified (Figure 1). This variant presumably induces nonsense-mediated mRNA decay due to an early stop codon leading to GATA6 haploinsufficiency [13].
GATA6: c.1291C >T p.(GIn431*)

\section{G C T T C T Y C G G C T T G}

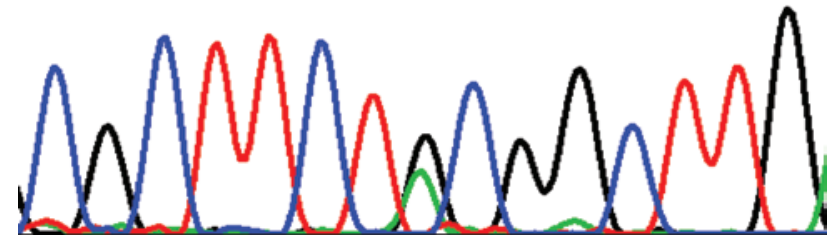

Figure 1: Electropherogram of the Sanger confirmation with the variant c. $1291 \mathrm{C}>\mathrm{T}$

\section{Discussion}

In our patient, the hyperglycemic episode in the neonatal period was of very short duration. At the time of the accidental diabetes diagnosis at the age of 3 years and initiation of insulin treatment, the patient showed no clinical symptoms of hyperglycemia. Therefore, no statement about the spontaneous progression of diabetes could be made. Interestingly, his insulin need has remained very low (0.2 E/kg body weight) so far.

Phenotypic consequences of the GATA6 variant appear to be stronger in the boy than in his mother: onset of diabetes was in childhood compared to adulthood in the mother. Moreover, the boy presented with diaphragmatic hernia and early-onset scoliosis, which were both missing in his mother. This matches the observations in other well-described autosomal-dominant inherited diseases in which patients with a mild phenotype are likely to have children with a more severe phenotype.

In the literature, the phenotypic spectrum of GATA6 variants comprises neonatal to adult onset of diabetes, no or small-to-complex congenital heart defects, and additional malformations such as diaphragmatic hernia and gallbladder agenesis, which are not constantly found in affected parents or siblings harboring the same variant $[8,9]$. We summarize 11 patients from the literature with hereditary cases of the GATA6 variant [8-11] in Table 1. In all these cases, a more severe phenotype could be observed in the second generation. In two of them, maternal mosaicism was described. Both mothers were without any symptoms (patient 10) or had just a small congenital heart defect (patient 6), whereas their children were severely affected. In all patients presented in Table 1, the onset of diabetes was earlier in the affected offspring if diabetes was present in the parents. In patients who presented with a congenital heart defect, some presented with a similar severity and some with 


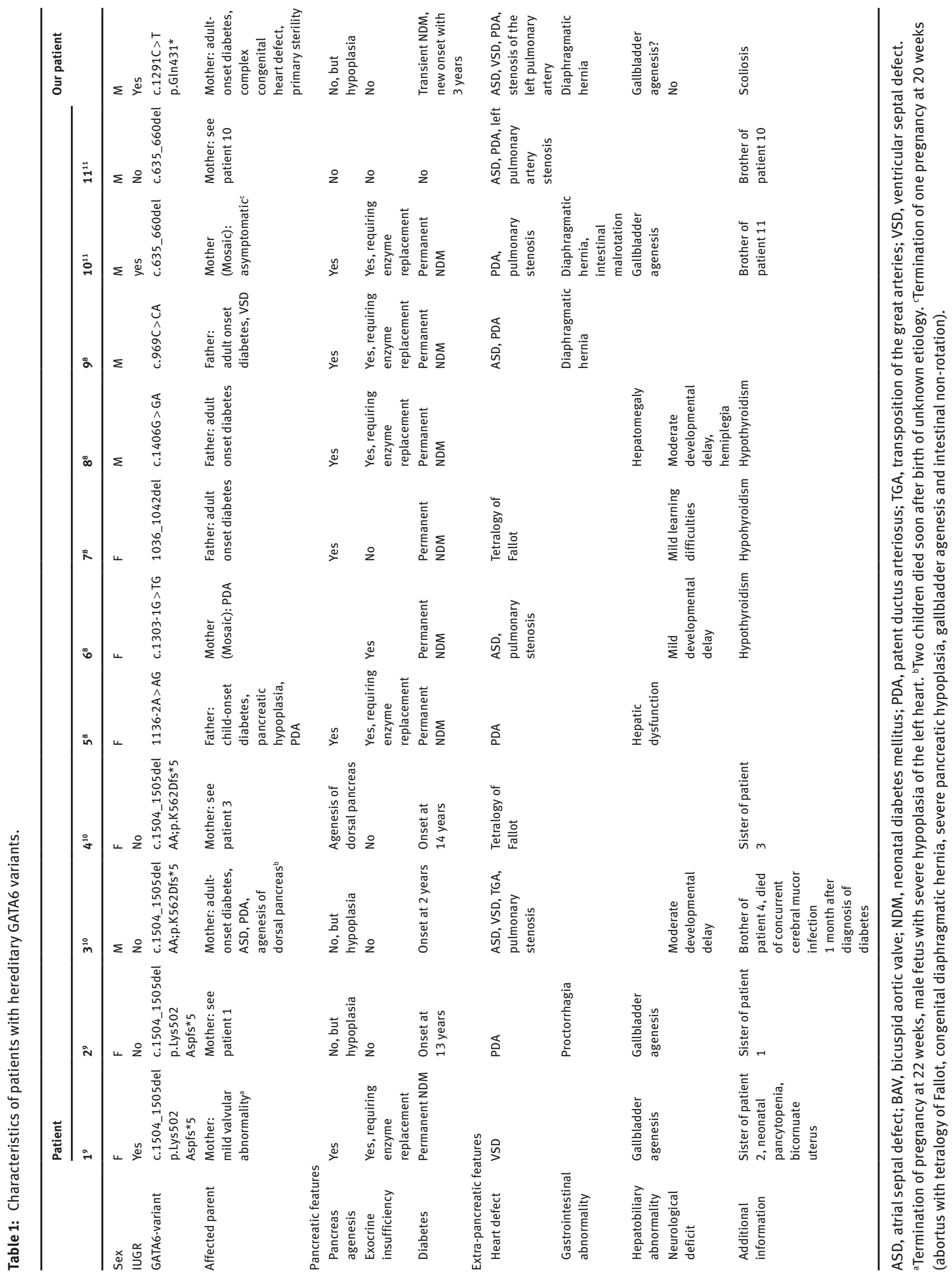


clear impairment of the cardiac malformation (patients 3, 4, 7, 10 and 11). Moreover, additional defects such as diaphragmatic hernia, scoliosis and hypothyroidism, which were inexistent in their affected parents, occurred in the second generation (patients 6, 7, 8, 9, 10 and our patient).

In conclusion, in our patient and in other patients with GATA6 variants from the literature, a variable phenotypic spectrum and also an increment in the severity of the phenotype from one generation to the next can be observed.

\section{Learning points}

GATA6 variants can be due to de novo variants or due to inherited variants.

GATA6 variants display a wide range of clinical phenotypes.

\section{What is new?}

A novel hereditary heterozygous GATA6 gene variant (c.1291C > T p.[Gln431*]) leading to neonatal diabetes, congenital heart defect and diaphragmatic hernia is described.

Patients with a mild phenotype are likely to have children with a more severe phenotype.

Acknowledgments: We thank the family of the patient for taking part in this research project.

Author contributions: OG and ML had full access to all of the data in the study and take responsibility for the integrity of the data and the accuracy of the data analysis. Study concept and design: OG, DK and ML. Acquisition, analysis or interpretation of data: All authors. Drafting of the manuscript: OG. Critical revision of the manuscript for important intellectual content: All authors. Administrative, technical or material support: DK, PJ and ML. Study supervision: ML.

Research funding: There was no funding for this research project.

Employment or leadership: None declared.

Honorarium: None declared.

Disclosure statement: The authors have no conflicts of interest to declare.
Ethical statement: The research was conducted ethically in accordance with the World Medical Association Declaration of Helsinki. The parents of our patient gave their informed consent to publish their case.

\section{References}

1. Laverriere AC, MacNeill C, Mueller C, Poelmann RE, Burch JB, et al. GATA-4/5/6, a subfamily of three transcription factors transcribed in developing heart and gut. J Biol Chem 1994;269:23177-84.

2. Gürson CT, Tahsinoglu M, Yakacikli S, Ertugrul T. A case of agenesis of the dorsal pancreas with interventricular septal defect in an infant. Helve Paediatr Acta 1970;25:522-6.

3. Von Mühlendahl KE, Herkenhoff H. Long-term course of neonatal diabetes. N Engl J Med 1995;333:704-8.

4. Aguilar Bryan L. Bryan J. Neonatal diabetes mellitus. Endocr Rev 2008;29:265-91.

5. Jeha GS, Venkatesh MP, Edelen RC, Kienstra KA, Karaviti L, et al. Neonatal diabetes mellitus: patient reports and review of current knowledge and clinical practice. J Pediatr Endocrinol Metab 2005;18:1095-102.

6. Iafusco D, Massa O, Pasquino B, Colombo C, lughetti L, et al. Early Diabetes Study Group of ISPED. Minimal incidence of neonatal/infancy onset diabetes in Italy is 1:90,000 live births. Acta Diabetol 2012;49:405-8

7. Molkentin JD. The zinc-finger-containing transcription factors GATA-4, -5 , and -6. Ubiquitously expressed regulators of tissue-specific gene expression. Biol Chem 2000;275: 38949-52.

8. De Franco E, Shaw-Smith C, Flanagan SE, Shepherd MH, Hatterslay AT, et al. GATA6 mutations cause a broad phenotypic spectrum of diabetes from pancreatic agenesis to adult-onset diabetes without exocrine insufficiency. Diabetes 2013;62:993-7.

9. Bonnefond A, Sand O, Guerin B, Duran E, De Graeve F, et al. GATA6 inactivating mutations are associated with heart defects and, inconsistently, with pancreatic agenesis and diabetes. Diabetologia 2012;55:2845-7.

10. Yorifuji T, Kawakita R, Hosokawa Y, Fujimaru R, Yamaguchi E, et al. Dominantly inherited diabetes mellitus caused by GATA6 haploinsufficiency: variable intrafamilial presentation. J Med Genet 2012;49:642-3.

11. Yau D, De Franco E, Flanagan S, Ellard S, Blumenkrantz M, et al. Case report: maternal mosaicism resulting in inheritance of a novel GATA6 mutation causing pancreatic agenesis and neonatal diabetes mellitus. Diagn Pathol 2017;12:1.

12. Allen HL, Flanagan SE, Shaw-Smith C, De Franco E, Akerman I, et al. GATA6 haploinsufficiency causes pancreatic agenesis in humans. Nat Genet 2011;44:20-2.

13. Suzuki S, Nakao A, Sarhat AR, Furuya A, Matsuo K, et al. A case of pancreatic agenesis and congenital heart defects with a novel GATA6 nonsense mutation: evidence of haploinsufficiency due to nonsense-mediated mRNA decay. Am J Med Genet A 2014;164:476-9. 